\title{
Research on Narrowband Interference Suppression and Diversity Characteristics in Fast Frequency-Hopping Communication Systems
}

\author{
Bing Zhao, Lei Xin, Jingyu Liu and Qun Ding \\ Electronic Engineering, HeilongjiangUniversity, Harbin,Heilongjiang, China \\ 150080 \\ Software School, Harbin Normal University, Harbin, Heilongjiang, China 150025 \\ 1zb0624@163.com \\ 2liujinyu926@163.com
}

\begin{abstract}
Frequency-hopping communication has the capability to resist narrowband interference, which is in direct proportion to the hopping rate. In frequency-hopping system, interference signals exist in partial de-hopping frequency-hopping signals, equivalent to time-varying interference signals existed in the frequency-hopping signals. Through integrating the diversity combining techniques of fast frequency-hopping communication with singular value decomposition method, this paper detects the frequency-hopping points, masks them, and then combines with undisturbed frequencyhopping signals. MATLAB simulation shows that narrowband interference is well restrained and the system BER is obviously improved.
\end{abstract}

Keywords: Fast Frequency-Hopping, Diversity Characteristics, Singular Value Decomposition, Narrowband Interference Suppression

\section{Introduction}

The current spread spectrum communication system can be divided into three types: direct sequence spread spectrum, frequency hopping and mixed type. Frequency-hopping (FH) communication can reach a fairly wide bandwidth and possess the performance of anti-interference, but when the intensity of interference signals far exceeds the anti-interference ability of FH communication system itself, the receiver will fail to receive data precisely, and decrease the anti-interference performance of frequency-hopping system. Therefore, scientists have proposed the interference suppression method to further enhance the anti-interference ability of frequency-hopping system [1-4].

Fast frequency-hopping (FFH) communication has a strong ability of antiinterference, but when the system requirement is very high, it will be hard for single frequency-hopping communication system to reach the performance requirements. For example, when the number of hopping is too large, it will increase the frequency bandwidth, and lead to low utilization rate of spectrum; when the channel spacing is narrowed, will lead to the hitting phenomenon, in which multiple users jump onto the same frequency simultaneously, will be likely to occur due to the drifting of oscillators and the indeterminateness of relative positions among transceivers, thus resulting in interference and error code. From the view of anti-interference, the larger number of frequency hopping, the interference smaller; but unduly large number of frequency hopping will make the system structure relatively complex. In addition, the frequency hopping bandwidth is usually restrained, so in a relatively long frequency-hopping pattern, the channels therein are often repeatedly used. In 
this way, if the delay of multi-path signals is very large, the collision of frequency will occur within specific time intervals, thus leading to error code $[5,6]$.

Singular value decomposition (SVD) method has been widely used in spectrum estimation in that its ability in distinguishing signals. The analysis of the singular value of received signals in autocorrelation matrix shows that the energy of interference signals is distributed in the singular value with large numerical value, while the energy of frequency-hopping signals is evenly distributed in characteristic space. It can be seen that SVD technique can obtain the required differentiation of signal characteristics [7].

This paper adopts the diversity combining property of fast frequency-hopping communication system and adds in the SVD method. Firstly, SVD method is used to detect interfered frequency-hopping signals, and signals interfered will be set zero; un-interfered ones will be de-hopping. Then all the processed signals are de-spread. In condition of SIR is large and channel environment is relatively good, the SVD algorithm does not improve anti-jamming capability significantly. When the interfering signals of continuous channels are much less than the diversity number, demodulation signals can acquire better anti-interference performance through SVD method.

\section{Fast Frequency-Hopping System Structure}

Fast frequency-hopping (FFH) refers to a hopping scenario in which multiple frequencies exist in the intervals of each modulation code. FFH technique boasts relatively good performance of anti-interference for its diversity combining feature. Figure 1 is the system structure diagram of FFH.

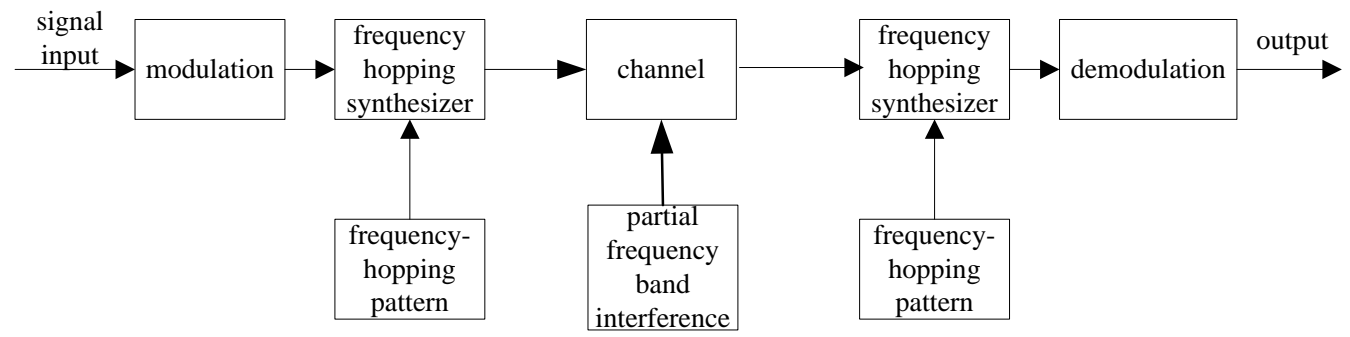

Figure 1. System Structure Diagram

In figure1, the frequency-hopping pattern serves as an important connection in FH communication. In FH communication, carrier frequency variations are called FH pattern. It is usually hoped that the rules of frequency hopping will not be fathomed out by enemies, so frequency-hopping pattern of pseudorandom variations are adopted. Only both communication sides know the frequency-hopping pattern, which are absolutely confidential to enemies. The pseudorandom means randomness is not true, in fact there are certain rules to be followed, but it is hard for enemies to guess out the rules without knowing the pattern [8].

After going through modulation, information signals are subjected to carrier modulation, which is produced by the frequency-hopping synthesizer that controlled by pseudorandom sequence. Going through carrier modulation, signals become frequency-hopping signals, which are to be received by receiver through channel transmission affected by broadband noise and single-frequency interference. The receiver firstly extracts the $\mathrm{FH}$ synchronous signals from the delivered modulation signals to make the frequency-hopping of local pseudorandom sequence control consistent with that of the received FH signals synchronized. In this way, local carriers with the same frequency as that of the transmitter are produced. Then it is 
demodulated by using local carriers and receiving signals to obtain signals bearing information.

The mathematical expression of frequency-hopping system is as follows:

$$
d(t)=\sum_{n=0}^{\infty} d_{n} g_{d}\left(t-n T_{d}\right)
$$

Among which, $d_{n}$ is the information code with a value of +1 and $-1 ; T_{d}$ is the width of information bit. As a rectangular function, $g_{d}$ can be expressed as:

$$
g_{d}(t)=\left\{\begin{array}{cc}
1 & -T_{d} \leq t \leq 0 \\
0 & 0 \leq t \leq T_{d}
\end{array}\right.
$$

Assume that the BPSK modulation is adopted and the frequency generated by the frequency synthesizer is $f_{i} \in\left\{f_{1} f_{2}, \cdots f_{N}\right\}$. Among which $f_{i}$ is one frequency of the value frequency set in $i T_{h} \leq t<(i+1) T_{h}$. It is controlled by pseudo codes, and $T_{h}$ stands for the duration of each hopping. Then the transmission signal obtained is:

$$
s(t)=d(t) \cos \left(2 \pi f_{i} t\right)
$$

Signal at the receiver is:

$$
r(t)=s(t)+n(t)+J(t)+S_{J}(t)
$$

$s(t)$ is useful signal, $n(t)$ is zero mean Gaussian white noise, $J(t)$ is narrowband interference signal, and $S_{J}(t)$ is the interference of other addresses in the channel. And it can be expressed as:

$$
S_{J}(t)=\sum_{\substack{j=1 \\ j \neq i}}^{k} d_{j}(t) \cos \left(2 \pi f_{j} t\right)
$$

Among which, $d_{j}(t)$ stands for the data of other users, and $k$ stands for the number of users in the channel. In this paper, assuming the vector sum of interference signals from other addresses is zero at the receiver, only study the suppression method of narrowband interference signal systematically.

Figure2 shows a frequency-hopping pattern, in which the horizontal axis is time and the vertical axis is frequency. The plane is called time-frequency domain. This domain can also be regarded as a chessboard, in which the time bands at horizontal axis and the frequency bands at vertical bands form the crisscrossed pattern. The asterisks represent the scheme of chess pieces, it is the frequency-hopping pattern, which indicates that different frequencies at different times are adopted to conduct communication.

The pseudo-random code which controls frequency hopping is generated by 8 shift register. As can be seen from the figure2, the system maximum frequency difference between adjacent frequency points is $128 \mathrm{MHz}$, the minimum frequency difference is $1 \mathrm{MHz}$, hopping repetition period is 255 . 


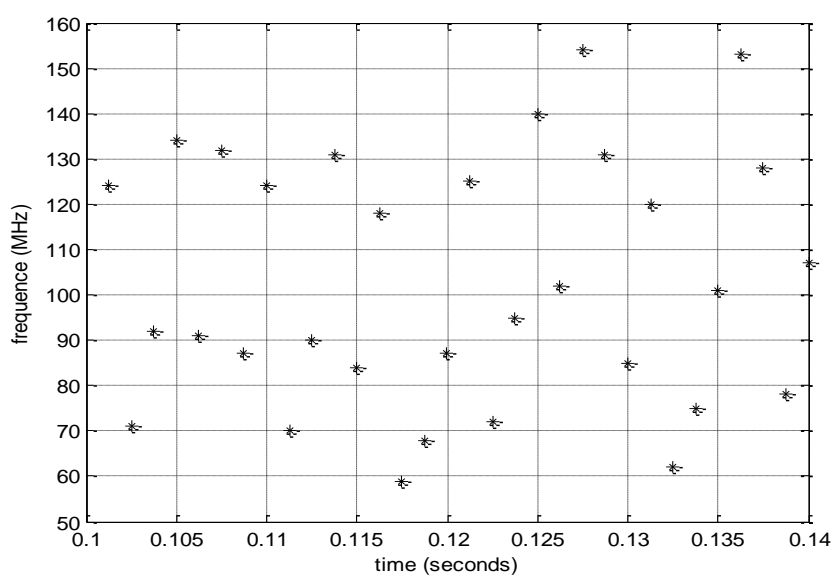

Figure 2. FFH Pattern

\section{Singular Value Decomposition}

As an effective way to deal with noise, SVD technique can be used to process signals polluted by additive noises. The definition of SVD is as follows: A stands for a matrix of $m \times n$ with the rank being $r \leq \min (m, n)$; there is orthogonal matrix $\boldsymbol{U}$ of $m \times m$ as well as orthogonal matrix $\boldsymbol{V}$ of $n \times n$ such that

$$
\boldsymbol{A}=\boldsymbol{U} \Sigma \boldsymbol{V}^{\mathrm{T}}
$$

Among which, $\Sigma$ stands for the diagonal matrix of $m \times n$ :

$$
\Sigma=\left[\begin{array}{ll}
\Delta & 0 \\
0 & 0
\end{array}\right], \quad \Delta=\operatorname{diag}\left(\sigma_{1}, \sigma_{2}, \cdots, \sigma_{r}\right)
$$

The column vectors of $\boldsymbol{U}$ and $\boldsymbol{V}$ are respectively the left and right singular value vectors of $\boldsymbol{A}$. And the $\sigma_{1}, \sigma_{2}, \cdots, \sigma_{r}$ is called the non-zero singular value of matrix $\boldsymbol{A}$.

When SVD is applied into signal processing, we need to construct a matrix $\boldsymbol{A}$ of $m \times n$ at first. Assume that the discrete sample data containing noise is

$$
r(n)=s(n)+n(n)
$$

The sampling interval is $\Delta t$, and $m$ segments, used for the $m$ lines of matrix $\boldsymbol{A}$, are intercepted at equal length of $n$ points from $r(n)$, then the matrix of $\boldsymbol{A}$ is constructed as follows:

$$
\boldsymbol{A}=\left[\begin{array}{cccc}
r(1) & r(2) & \cdots & r(n) \\
r(n+1) & r(n+2) & \cdots & r(2 n) \\
\vdots & \vdots & \vdots & \vdots \\
r((m-1) n+1) & r((m-1) n+2) & \cdots & r(m n)
\end{array}\right]=\boldsymbol{A}_{s}+\boldsymbol{W}
$$

Where $\boldsymbol{A}_{s}$ stands for the matrix of signal composition, $\boldsymbol{W}$ stands for the matrix composed of noises, with $\boldsymbol{A}_{s} \in \mathrm{R}^{m \times n}$ and $\boldsymbol{W} \in \mathrm{R}^{m \times n}$.

In fact, SVD calculation for $r(n)$ is the process of seeking the best approximating value of $\boldsymbol{A}_{s}$ on the basis of $\boldsymbol{A}$. The higher the approximating level, the better the denoising performance. For the matrix $\boldsymbol{A}_{s}$ composed of signals, the row vectors have 
correlation degree, and the rank of matrix is 1 . According to the theory of SVD, the number of non-zero singular value is equal to the rank of matrix, but to the numerical calculation of SVD, no singular value can reach zero, thus here the "non-zero singular value" can be understood as a value larger than a very small positive number $\varepsilon$.

The row vectors in matrix of $W$ areirrelevant. Besides, the rank of the matrix is $m$ with similar singular values.

$$
\sigma(\boldsymbol{W})=(\xi, \xi, \cdots, \xi)
$$

The singular value of matrix $\boldsymbol{A}_{s}$ and $\boldsymbol{W}$ against the matrix of $\boldsymbol{A}$ conforms to the following formula:

$$
\sigma(\boldsymbol{A}) \leq \sigma\left(\boldsymbol{A}_{s}\right)+\sigma(\boldsymbol{W})
$$

Here, the non-zero singular value of matrix $\boldsymbol{A}$ contains the concentrated energy situation of signals and noises in sampled data. If there is no noise in signal or the SNR is high, $r<\min (m, n)$; if there is noise in signal or the SNR ratio is low, $r=\min (m, n)$. The top $k$ relatively large singular values in non-zero singular values mainly reflect signals, while relatively small ones reflect noises. The singular values reflecting noise are set to zero, and only the top $k$ relatively large singular values are kept. Then through the reverse transformation of SVD, the approximation matrix $\hat{\boldsymbol{A}}$ is rebuilt, in which the noises are reduced. When $r(n)$ only contains periodic signals with a cycle of $T$ without noise and $n \Delta t=k T(k=0,1,2,3 \mathrm{~L})$, then only one non-zero singular value $\sigma_{1}$ is obtained through SVD. Under other cases, more than one non-zero singular values are obtained. When an integral value near $k T / \Delta t$ is assigned to $M$, then $\sigma_{1}$ will be much larger than other singular values.

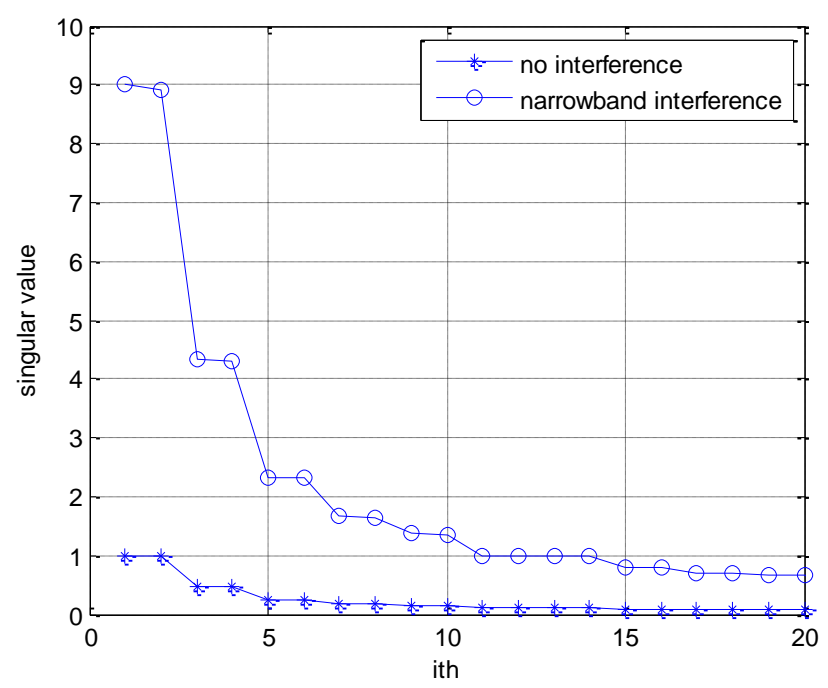

\section{Figure 3. Singular Value of Signals on Interfered Frequency Point and Un- Interfered Frequency Point}

Figure 3 shows the first 20 order singular values of interfered frequency point signal and un-interfered frequency point signal. Assuming the center frequency of narrowband interference signal overlaps frequency hopping point completely, and the interference signal bandwidth is equal to the instantaneous system bandwidth, that is the whole band of hopping frequency point is interfered.The SIR is -20dB. For analytical purposes, assuming largest singular value $\sigma_{1}$ of undisturbed frequency 
point signal is 1 , then the $\sigma_{1}$ of interfered frequency point signal is 9 times of it. Due to the presence of narrowband interference, the singular values increase significantly.

Table 1 is first singular values on consecutive 6 hopping frequency points. After normalized treatment, it can be seen two larger singular values, there are interference signal on $f_{i-1}$ and $f_{i+2}$. In receiver, the signal of these two frequencies will be set to 0 , that is the disturbed signal is discarded, using other diversity signal to merge.

Table 1. First Singular Values on Different Hopping Frequency Point

\begin{tabular}{c|c|c|c|c|c|c}
\hline $\begin{array}{c}\text { Hopping frequency } \\
\text { point }\end{array}$ & $f_{i-2}$ & $f_{i-1}$ & $f_{i}$ & $f_{i+1}$ & $f_{i+2}$ & $f_{i+3}$ \\
\hline $\begin{array}{c}\text { First singular } \\
\text { value } \sigma_{1}\end{array}$ & 1 & 11.88 & 1.002 & 1 & 11.59 & 1.004 \\
\hline
\end{tabular}

\section{Narrowband Interference Suppression Based on FFH Combined with SVD}

In FFH method, due to the fact that the spread spectrum signals of each bit will choose several FH frequency points to transmit data, the remaining un-disturbed FH signals can still precisely demodulate the bit information even though partial signals are influenced by interference, and finally the information going through dispreading will be sent into the diversity combining module.

Researches on the anti-interference performance of FFH are mainly focused on the diversity combining algorithm under the environment of interference. Diversity means diverting a signal to different channels for transmission. When certain subchannels undergo external interference in transmission, the receiver can receive the information of the other channels containing the signal segments, and restore the information through relevant information observation.

When spread spectrum signals are interfered, the singular values are uneven in SVD operation. So a threshold needs to be set, when the characteristic value is larger than the threshold, it will be determined as invalid signal. Combined with advantage of the diversity characteristics of $\mathrm{FFH}$, un-interfered signals are combined after SVD detection. Then after the processes of dispreading and restoring, original data is obtained. Figure4 is the narrowband interference suppression on the basis of FFH combined with SVD.

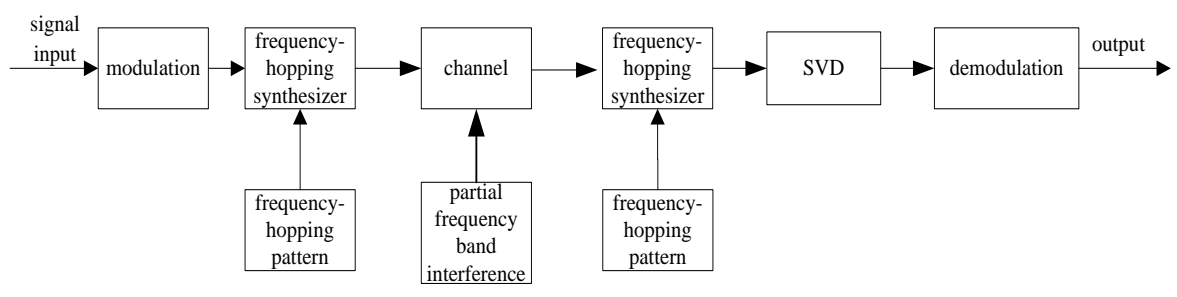

Figure 4. FFH System Combined with SVD

\section{Simulation Result Analysis}

Assuming deliver 10000 bits of information, information rate $R_{b}$ is 200bit/s, and the PN code is the $\mathrm{m}$ sequence, the number of frequency hopping points is 256. Under the conditions that the hopping rate of SFH is $1 \mathrm{bit} / \mathrm{hop}$ and the hopping rate 
of FFH is 1 bit/4hop, the interference model falls into narrowband interference, the number of disturbed frequency points is $20 \%$ of the available frequency points. Figure 5 is the curve graph of SNR and BER under the condition of no narrowband interference. Figure6 is the same curve graph under the condition of narrowband interference, and the SIR ratio is $-15 \mathrm{~dB}$.

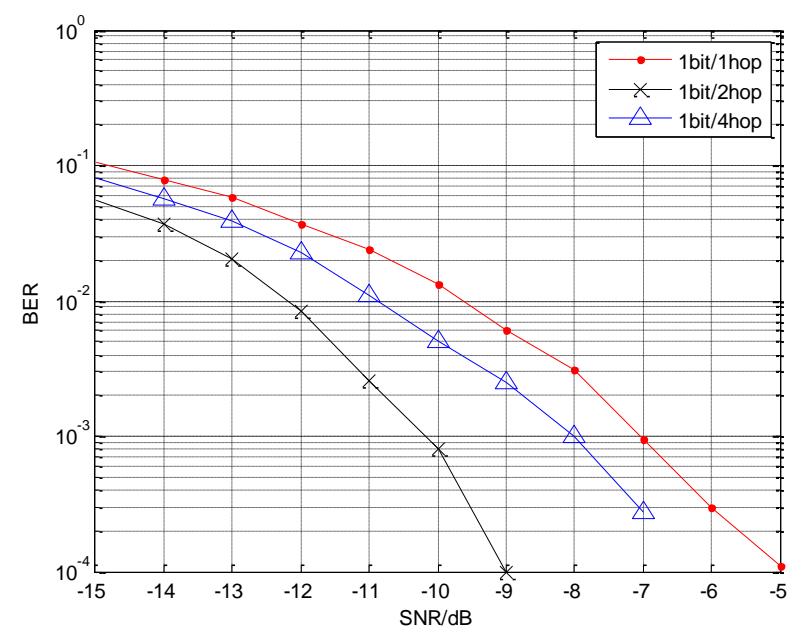

Figure 5. Curve graph of SNR and BER under the Condition of No Narrowband Interference

As is shown in Figure5, when the hopping rate is relatively low, the ability of interference suppression is limited, with the speeding up of hopping rate, the BER declines.

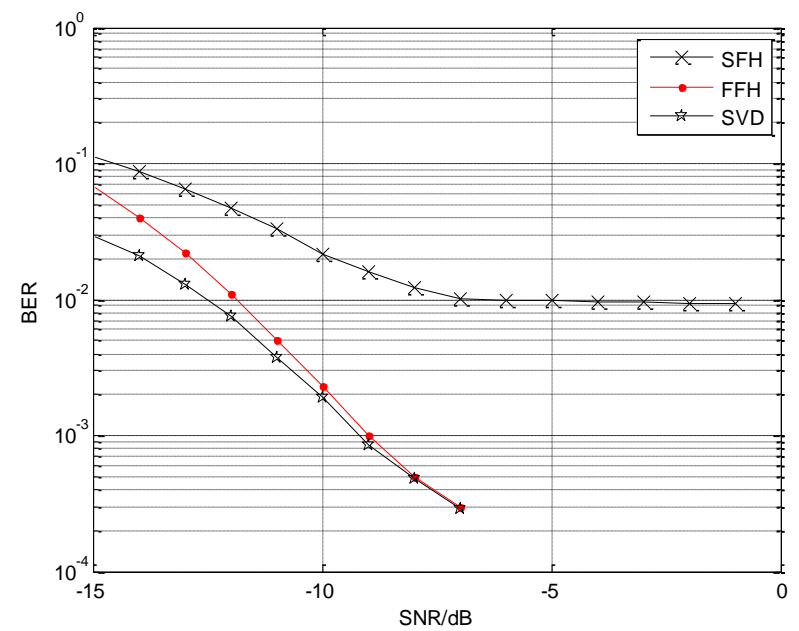

Figure 6. Curve Graph of SNR and BER under the Condition of Narrowband Interference

Figure6 shows the curve graph of narrowband interference suppression performance. Narrowband interference exerts randomly influences on hopping frequency points. It can be seen from the graph, for slow frequency hopping system, when the SNR is greater than $-7 \mathrm{~dB}$, narrowband interference effects become apparent, the BER is not reduced with the increase of SNR, the system maintains a high BER. For FFH system without SVD, when the SNR is greater than $-12 \mathrm{~dB}$, the role of narrowband interference began to highlight. For the system combined SVD 
and FFH, narrowband interference has been suppressed in a manner, especially in the case of low SNR. With the increase of SNR, system BER closes to zero.

\section{Conclusion}

In this paper, the FFH communication system judge received signals whether or not be interfered according to SVD method firstly, then using the diversity combining characteristic, the interfered FH signals are eliminated and the uninterfered FH signals are used for de-hopping. Through system simulation, it can be seen that in case of a strong narrowband noise, SVD can be adopted to detect whether or not signals are interfered effectively, with a view to obtaining ideal effect of interference suppression.

\section{Acknowledgements}

This work is supported by Heilongjiang Provincial Education Department Science and Technology Research Project (NO.12531492), Heilongjiang Provincial Department of education project (based on multi level classification and early warning of building intelligent monitoring system 12541237); Young academic foundation of Harbin Normal University (KGB201215).Many thanks to the anonymous reviewers, whose insightful comments made this a better paper.

\section{References}

[1] J. J. Perez-Slolano, S. Feliei-Castell and M. A. Rodriguez-Hcrndndez, "J. Narrowband Interference Suppression in Frequency-Hopping Spread Spectrum Using Undecimated Wavelet Packet Transform", IEEE Transactions on Vehicular Technology, vol. 3, no. 57 (2008), pp. 1620-1628

[2] W. M. Saad and I. Marsland, "Editors, Weighted Random Frequency Hopping in the Presence of Narrowband Interference", Proceedings of Electrical Engineering/Electronics, Computer, Telecommunications and Information Technology, (2013), pp. 1-5, Krabi.

[3] R. Zhi, L. Zhang and Z. Zhou, "Edtors, Cognitive Frequency Hopping", Proceedings of Cognitive Radio Oriented Wireless Networks and Communications, (2008), pp. 1-4, Singapore.

[4] L. M. D. Le, K. C. Teh, K. H. J. Li, "Survey on diversity combining techniques for interference suppression in fast frequency hopping systems", Communications, IET, vol. 12, no. 9, (2015), pp. 15011509.

[5] T. Li, Q. Ling and J. Ren, "Editors, Spectrally Efficient Frequency Hopping System Design for Wireless Network", Proceedings of Wireless Algorithms, Systems and Applications, (2007), pp. 244-248, Chicago.

[6] T. T. Nguyen, H. H. Nguyen and T. Le-Ngoc, "J, Iterative interference cancellation in multiuser relaying with fast frequency-hopping modulation", Communications, IET, vol. 15, no. 8, (2014), pp. 2693-2705.

[7] L. B. Milstein, "J, Interference rejection techniques in spread spectrum communications", Proc, IEEE, vol. 6, no. 76, (1988), pp. 657-671.

[8] X. Zhao and X. Kang, "Editors, The Study of the Spread Spectrum Communication System Based on Sequence Pairs", Proceedings of Communications and Information Technology, (2005), pp. 548-551.

Authors

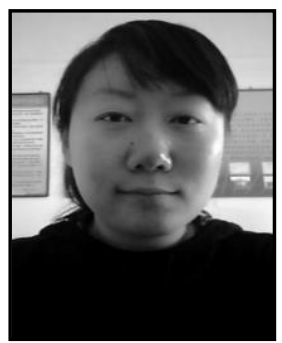

Bing Zhao, the Han nationality, native place: Hebei, doctor's degree, principal research directions: broadband communications, digital signal processing, E-mail:zb0624@163.com 